\title{
Maximal perpendicularity in certain Abelian groups
}

\author{
Mika Mattila \\ Department of Mathematics, Tampere \\ University of Technology, Finland \\ email: mika.mattila@tut.fi \\ Pentti Haukkanen \\ School of Information Sciences, \\ University of Tampere, Finland \\ email: pentti.haukkanen@uta.fi
}

\author{
Jorma K. Merikoski \\ School of Information Sciences, \\ University of Tampere, Finland \\ email: jorma.merikoski@uta.fi \\ Timo Tossavainen \\ Department of Art, Communication \\ and Education, \\ Lulea University of Technology, Sweden \\ email: timo.tossavainen@ltu.se
}

\begin{abstract}
We define perpendicularity in an Abelian group $\mathrm{G}$ as a binary relation satisfying certain five axioms. Such a relation is maximal if it is not a subrelation of any other perpendicularity in G. A motivation for the study is that the poset $(\mathcal{P}, \subseteq)$ of all perpendicularities in $\mathrm{G}$ is a lattice if $\mathrm{G}$ has a unique maximal perpendicularity, and only a meet-semilattice if not. We study the cardinality of the set of maximal perpendicularities and, on the other hand, conditions on the existence of a unique maximal perpendicularity in the following cases: $G \cong \mathbb{Z}^{n}, G$ is finite, $G$ is finitely generated, and $G=\mathbb{Z} \oplus \mathbb{Z} \oplus \cdots$. A few such conditions are found and a few conjectured. In studying $\mathbb{R}^{n}$, we encounter perpendicularity in a vector space.
\end{abstract}

\section{Introduction}

Over the years, the concept of "perpendicular" has been considered axiomatically from several different perspectives. Perhaps the most well-known axiomatic description of perpendicularity is presented in the classical textbook 
[1] by Bachmann. This approach is designed for the construction of plane geometry and it is based on studying reflections in the metric plane which is a notion to serve as a common basis of Euclidean, hyperbolic and elliptic planes.

Davis $[2,3]$ studied rings and Abelian groups with orthogonality relations. In his approach, the aim of defining an orthogonality relation on an Abelian group was to generalize the concept of a disjointness relation on a linear space introduced earlier by Veksler [7].

A more recent axiomatization of perpendicularity and parallelism is given in [4]. This axiom system was originally constructed for educational purposes and it is applicable enough for the examination of the geometry of perpendicular and parallel lines in the Euclidean plane, and certain other non-trivial planar or numeric models, too.

The present approach to defining algebraic perpendicularity was originally laid down in [5]; this article is a sequel to that. Our definition is based on the idea of describing the additive properties of the elements of an inner product space for which the inner product is zero in terms of the binary operation of an Abelian group.

Following the notation of [5], let $\mathrm{G}=(\mathrm{G},+)$ be an Abelian group, $\mathrm{G} \neq\{0\}$, and let $\perp$ be a perpendicularity in $\mathrm{G}$, that is, a binary relation satisfying

(A1) $\forall a \in G: \exists b \in G: a \perp b$,

(A2) $\forall \mathrm{a} \in \mathrm{G} \backslash\{0\}: \mathrm{a} \not \perp a$,

(A3) $\forall \mathrm{a}, \mathrm{b} \in \mathrm{G}: \mathrm{a} \perp \mathrm{b} \Rightarrow \mathrm{b} \perp \mathrm{a}$,

(A4) $\forall \mathrm{a}, \mathrm{b}, \mathrm{c} \in \mathrm{G}: \mathrm{a} \perp \mathrm{b} \wedge \mathrm{a} \perp \mathrm{c} \Rightarrow \mathrm{a} \perp(\mathrm{b}+\mathrm{c})$,

(A5) $\forall \mathrm{a}, \mathrm{b} \in \mathrm{G}: \mathrm{a} \perp \mathrm{b} \Rightarrow \mathrm{a} \perp-\mathrm{b}$.

The trivial perpendicularity

$$
\mathrm{a} \perp \mathrm{b} \Longleftrightarrow \mathrm{a}=0 \vee \mathrm{b}=0
$$

always exists. A perpendicularity $\perp$ is minimal if it is not a superrelation of any other perpendicularity in G. This clearly happens if and only if $\perp$ is trivial; hence, minimal perpendicularity is always unique. Similarly, a perpendicularity is maximal if it is not a subrelation of any other perpendicularity in G.

A few results on minimal and maximal perpendicularities follow easily.

Proposition 1 If $\mathrm{G}$ is cyclic, then it has a unique maximal perpendicularity. If $\mathrm{G}$ is cyclic and infinite, then it has only the trivial perpendicularity. 
Proof. See [5, Theorem 14] and [5, Example 8].

Maximal perpendicularity is not necessarily unique even if $G$ is finite. For example [5, Example 7], the Klein four group has three nontrivial perpendicularities, all of them maximal.

Proposition 2 A maximal perpendicularity always exists.

Proof. If $\perp_{1} \subseteq \perp_{2} \subseteq \ldots$ are perpendicularities in $G$, then $\cup_{i=1}^{\infty} \perp_{i}$ is clearly a perpendicularity in G. So, the claim follows from Zorn's lemma.

Let $(\mathcal{P}, \subseteq$ ) be the poset (partially ordered set) of all perpendicularities in $\mathrm{G}$. (In fact, every nonempty family of sets is a poset under subset relation.)

Proposition 3 A perpendicularity in $\mathrm{G}$ is maximal if and only if it is a maximal element of $\mathcal{P}$. There is a unique maximal perpendicularity in $\mathrm{G}$ if and only if there is a largest element in $\mathcal{P}$. The trivial perpendicularity is the unique minimal perpendicularity of $\mathrm{G}$, in other words, the smallest element of $\mathcal{P}$.

Proof. Easy and omitted.

A motivation for the present study is that $\mathcal{P}$ is a lattice if $G$ has a unique maximal perpendicularity, and only a meet-semilattice if not. Below we survey the uniqueness of maximal perpendicularity in the following cases: $G \cong \mathbb{Z}^{n}$ (Section 2), $\mathrm{G}$ is finite (Section 3), $\mathrm{G}$ is finitely generated (Section 4), and $\mathrm{G} \cong \mathbb{Z} \oplus \mathbb{Z} \oplus \cdots \cong\left(\mathbb{Q}_{+}, \cdot\right)$ (Sections 5 and 6 ). In addition to solving the question about the uniqueness in certain cases, we shall conjecture a few equivalent conditions for the existence of a unique maximal perpendicularity. We complete our paper by regarding $\mathbb{R}^{n}$ both as an additive group and as a vector space.

\section{$2 \quad \mathrm{G} \cong \mathbb{Z}^{\mathrm{n}}, \mathrm{n}>1$}

If $G \cong \mathbb{Z}$, then it has only the trivial perpendicularity by Proposition 1 . The case of $G \cong \mathbb{Z}^{n}=\mathbb{Z} \oplus \cdots \oplus \mathbb{Z}$ ( $n$ copies, $\left.n>1\right)$ is hence more interesting.

Let us choose $g_{1}, \ldots, g_{n} \in G$ such that

$$
\begin{aligned}
g_{1} & =(1,0,0,0, \ldots, 0), \\
g_{2} & =\left(\gamma_{21}, 1,0,0, \ldots, 0\right), \\
g_{3} & =\left(\gamma_{31}, \gamma_{32}, 1,0, \ldots, 0\right), \\
\vdots & \\
g_{n} & =\left(\gamma_{n 1}, \gamma_{n 2}, \ldots, \gamma_{n \cdot n-1}, 1\right),
\end{aligned}
$$


where the $\gamma_{i j}$ 's are integers. Denote by $\langle\cdot\rangle$ the generated subgroup.

Lemma 1 If $\mathrm{G} \cong \mathbb{Z}^{\mathrm{n}}$ and $\mathrm{g}_{1}, \ldots, \mathrm{g}_{\mathrm{n}}$ are as in $(1)$, then

$$
\mathrm{G}=\left\langle\mathrm{g}_{1}\right\rangle \oplus \cdots \oplus\left\langle\mathrm{g}_{\mathfrak{n}}\right\rangle \text {. }
$$

Proof. For any $x \in G$, there obviously are unique $\xi_{1}, \ldots, \xi_{n} \in \mathbb{Z}$ satisfying

$$
x=\xi_{1} g_{1}+\cdots+\xi_{n} g_{n} .
$$

Let $g_{1}, \ldots, g_{\mathfrak{n}}, n>1$, be as above. Also choose $g_{1}^{\prime}, \ldots, g_{\mathfrak{n}}^{\prime} \in G$ as in (1) such that $g_{i}^{\prime} \neq g_{i}$ for at least one $i \in N=\{1, \ldots, n\}$. So, there is $m \in N$ with

$$
g_{1}=g_{1}^{\prime}, \ldots, g_{m-1}=g_{m-1}^{\prime}, g_{m} \neq g_{m}^{\prime} \text {. }
$$

Let $a, b \in \mathrm{G}$. Then, by Lemma 1 ,

$$
a=a_{1}+\cdots+a_{n}=a_{1}^{\prime}+\cdots+a_{n}^{\prime}, \quad b=b_{1}+\cdots+b_{n}=b_{1}^{\prime}+\cdots+b_{n}^{\prime},
$$

where $a_{i}, b_{i} \in\left\langle g_{i}\right\rangle$ and $a_{i}^{\prime}, b_{i}^{\prime} \in\left\langle g_{i}^{\prime}\right\rangle$ for all $i \in N$.

Define now the relations $\perp_{0}$ and $\perp_{0}^{\prime}$ by

$$
\begin{aligned}
& a \perp_{0} b \Longleftrightarrow \forall i \in N: a_{i}=0 \vee b_{i}=0, \\
& a \perp_{0}^{\prime} b \Longleftrightarrow \forall i \in N: a_{i}^{\prime}=0 \vee b_{i}^{\prime}=0 .
\end{aligned}
$$

These relations are clearly perpendicularities in $\mathrm{G}$.

Lemma 2 Let $\perp_{0}$ and $\perp_{0}^{\prime}$ be as in (5). A maximal perpendicularity $\perp_{\max }$ in $\mathrm{G} \cong \mathbb{Z}^{\mathrm{n}}, \mathrm{n}>1$, cannot contain both of them.

Proof. We proceed by contradiction. Suppose that

$$
\perp_{\max } \supseteq \perp_{0} \cup \perp_{0}^{\prime} \text {. }
$$

We have $g_{m} \perp_{0} g_{1}, \ldots, g_{m-1}$ and $g_{m}^{\prime} \perp_{0}^{\prime} g_{1}^{\prime}, \ldots, g_{m-1}^{\prime}$ implying that $g_{m}^{\prime} \perp_{0}^{\prime}$ $g_{1}, \ldots, g_{m-1}$ by $(3)$. Therefore

$$
g_{m}, g_{m}^{\prime} \perp_{\max } g_{1}, \ldots, g_{m-1}
$$

by (6). Now, applying (A3), (A4) and (A5) yields that

$$
\left(g_{m}-g_{m}^{\prime}\right) \perp_{\max }\left(\xi_{1} g_{1}+\cdots+\xi_{m-1} g_{m-1}\right)
$$

for all $\xi_{1}, \ldots, \xi_{m-1} \in \mathbb{Z}$.

But $d=g_{m}-g_{m}^{\prime}=\left(\delta_{1}, \ldots, \delta_{\mathfrak{n}}\right)$ has $\delta_{m}=\cdots=\delta_{n}=0$, which implies that there are $\xi_{1}, \ldots, \xi_{m-1} \in \mathbb{Z}$ such that $d=\xi_{1} g_{1}+\cdots+\xi_{m-1} g_{m-1}$. So, $d \perp_{\max } d$ violating (A2) because $d \neq 0$ by (3). 
Theorem 1 There are infinitely many maximal perpendicularities in $\mathrm{G} \cong \mathbb{Z}^{\mathfrak{n}}$, $n>1$.

Proof. There are infinitely many choices of the $g_{i}$ 's in (1). Different choices give different $\perp_{0}$ 's in (5). Hence, the claim follows from Lemma 2.

Is $\perp_{0}$ defined by (5) maximal? The answer is negative. Namely, let $a, b \in G$ and write them as

$$
a=\alpha_{1} g_{1}+\cdots+\alpha_{n} g_{n}, \quad b=\beta_{1} g_{1}+\cdots+\beta_{n} g_{n},
$$

where the $\alpha_{i}$ 's and $\beta_{i}$ 's are integers. Define $\perp_{1}$ by

$$
\mathrm{a} \perp_{1} \mathrm{~b} \Longleftrightarrow \alpha_{1} \beta_{1}+\cdots+\alpha_{\mathrm{n}} \beta_{\mathrm{n}}=0 .
$$

Obviously $\perp_{1}$ is a perpendicularity and $\perp_{0}$ is its proper subset. But then, is $\perp_{1}$ maximal? This question remains open, yet we conjecture as follows.

Conjecture 1 A perpendicularity in $\mathrm{G} \cong \mathbb{Z}^{\mathrm{n}}, \mathrm{n}>1$, is maximal if and only if it is of the form (7).

We encounter another open question concerning the cardinality of the set $S$ of maximal perpendicularities in $\mathrm{G} \cong \mathbb{Z}^{\mathrm{n}}, \mathrm{n}>1$. Denoting by $|\cdot|$ the cardinality, Theorem 1 yields that $|S| \geq \aleph_{0}$. On the other hand, $\left|\mathbb{Z}^{\mathfrak{n}} \times \mathbb{Z}^{\mathfrak{n}}\right|=\aleph_{0}$, so, the cardinality of the set of all binary relations in $G \cong \mathbb{Z}^{\mathfrak{n}}$ is $2^{\mathrm{x}_{0}}$. Consequently, $|S| \leq 2^{\mathrm{N}_{0}}$.

But which of these inequalities is equality? The following proposition tells what we already know.

Proposition 4 If Conjecture 1 is true, then the set of maximal perpendicularities in $\mathrm{G} \cong \mathbb{Z}^{\mathrm{n}}, \mathrm{n}>1$, has cardinality $\boldsymbol{\aleph}_{0}$.

Proof. A maximal perpendicularity is of the form (5) by the conjecture. Because there are countably infinite choices of each $g_{i}, i>1$, in (1), there are also countably infinite choices of the sequence $\left(g_{1}, \ldots, g_{n}\right)$.

\section{Finite G}

In this section, we assume that $\mathrm{G}$ is also finite (in addition to being Abelian). If $\mathrm{G}$ is cyclic, then it has a unique maximal perpendicularity by Proposition 1 . So, in the rest of this section, suppose that $G$ be noncyclic if not mentioned otherwise. We begin by describing its structure. 
Theorem 2 If $\mathrm{G}$ is noncyclic and finite, then it has cyclic subgroups $\mathrm{H}_{1}, \ldots, \mathrm{H}_{\mathrm{r}}$, $r>1$, of prime power order such that

$$
\mathrm{G}=\mathrm{H}_{1} \oplus \cdots \oplus \mathrm{H}_{\mathrm{r}}
$$

These orders are unique. All decompositions (8) have the same number of summands of each order.

Proof. See [6, p. 394, Theorem 1].

Let $a, b \in G$, and let $H_{1}, \ldots, H_{r}$ be as in (8). Analogously to (4),

$$
a=a_{1}+\cdots+a_{r}, \quad b=b_{1}+\cdots+b_{r},
$$

where $a_{i}, b_{i} \in H_{i}$ for all $i=1, \ldots, r$. Similarly as in (5), we now define

$$
a \perp_{0} b \Longleftrightarrow \forall i \in\{1, \ldots, r\}: a_{i}=0 \vee b_{i}=0 .
$$

Further, if $\emptyset \neq A, B \subseteq G$, we write $A \perp B$ denoting that $x \perp y$ for all $x \in A$, $y \in B$.

Lemma 3 Let $\perp$ be a perpendicularity in $\mathrm{G}$, and let $\mathrm{a}, \mathrm{b} \in \mathrm{G}$. If $\mathrm{a} \perp \mathrm{b}$, then $\langle\mathrm{a}\rangle \perp\langle\mathrm{b}\rangle$ and $\langle\mathrm{a}\rangle \cap\langle\mathrm{b}\rangle=\{0\}$.

Proof. Let $\xi, \eta \in \mathbb{Z}$. Then $a \perp \eta b$ by (A4) and (A5). Further, applying also (A3), we get $\xi a \perp \eta b$. This proves the first claim. If $z \in\langle a\rangle \cap\langle b\rangle$, then $z=\xi a=\eta b$ for some $\xi, \eta \in \mathbb{Z}$. Now, the first claim implies that $z \perp z$; hence, $z=0$ by (A1) verifying the second claim.

Theorem 3 Let $\mathrm{G}$ be noncyclic and finite. If $|\mathrm{G}|$ is square-free, then $\mathrm{G}$ has a unique maximal perpendicularity which, in fact, is $\perp_{0}$ defined in (9).

Proof. Let $\perp$ be a perpendicularity in G. We claim that $\perp \subseteq \perp_{0}$. We can omit the trivial perpendicularity; so, we suppose that $0 \neq x, y \in G$ and $x \perp y$.

By Theorem 2 and square-freeness, $G$ has cyclic subgroups $\mathrm{H}_{1}, \ldots, \mathrm{H}_{\mathrm{r}}$ with prime orders $p_{1}, \ldots, p_{r}, r>1$, respectively, such that

$$
\mathrm{G}=\mathrm{H}_{1} \oplus \cdots \oplus \mathrm{H}_{\mathrm{r}} .
$$

Clearly,

$$
H_{i}=\left\{x \in G|| x \mid=p_{i}\right\}, \quad i=1, \ldots, r,
$$


where $|\cdot|$ denotes the order. Hence, this decomposition is unique (up to the ordering). Therefore, if $\mathrm{H}$ is a subgroup of $\mathrm{G}$, then

$$
\mathrm{H}=\mathrm{H}_{\mathrm{t}_{1}} \oplus \cdots \oplus \mathrm{H}_{\mathrm{t}_{\mathrm{s}}}
$$

for certain indices $t_{1}, \ldots, t_{s} \in\{1, \ldots, r\}$. In particular, there are indices $i_{1}, \ldots, i_{k}$ and $j_{1}, \ldots, j_{l}$ such that

$$
\langle x\rangle=H_{i_{1}} \oplus \cdots \oplus H_{i_{k}}, \quad\langle y\rangle=H_{j_{1}} \oplus \cdots \oplus H_{j_{l}} .
$$

Since $\langle x\rangle \cap\langle y\rangle=\{0\}$ by Lemma 3 , we have $\mathrm{H}_{\mathfrak{i}_{u}} \neq \mathrm{H}_{j_{v}}$ for all $u, v$. Therefore, $x \perp_{0} y$, and the claim follows.

We conjecture that also the converse holds.

Conjecture 2 Let $\mathrm{G}$ be as in Theorem 2. The following conditions are equivalent:

(a) G has a unique maximal perpendicularity,

(b) $|\mathrm{G}|$ is square-free,

(c) (8) is unique (up to the ordering of the $\mathrm{H}_{i}$ 's).

Theorem 3 states that $(\mathrm{b}) \Rightarrow(\mathrm{a})$. The following proposition states that $(\mathrm{a}) \Rightarrow(\mathrm{c})$. The part $(\mathrm{c}) \Rightarrow(\mathrm{b})$ remains open.

Proposition 5 Let $\mathrm{G}$ be as above. If $\mathrm{G}$ has a unique maximal perpendicularity, then (8) is unique.

Proof. Contrary to the uniqueness of (8), we suppose that there are decompositions

$$
\mathrm{G}=\mathrm{H}_{1} \oplus \cdots \oplus \mathrm{H}_{\mathrm{r}}=\mathrm{H}_{1}^{\prime} \oplus \cdots \oplus \mathrm{H}_{\mathrm{r}}^{\prime}
$$

such that $\left\{H_{1}, \ldots, H_{r}\right\} \neq\left\{H_{1}^{\prime}, \ldots, H_{r}^{\prime}\right\}$. Let $H_{i}=\left\langle g_{i}\right\rangle$ and $H_{i}^{\prime}=\left\langle g_{i}^{\prime}\right\rangle, i=$ $1, \ldots, r$. We define $\perp_{0}$ as we did in (9) and $\perp_{0}^{\prime}$ in an analogous manner applying $\mathrm{G}=\mathrm{H}_{1}^{\prime} \oplus \cdots \oplus \mathrm{H}_{\mathrm{r}}^{\prime}$. As in the proof of Lemma 2, we can show that no maximal perpendicularity contains both $\perp_{0}$ and $\perp_{0}^{\prime}$. (In this lemma, $g_{1}=g_{1}^{\prime}$, but without any role in the proof.) The uniqueness of maximal perpendicularity is thus violated. 


\section{Finitely generated G}

Next, we assume that $\mathrm{G}$ is finitely generated. In Proposition 1 and Theorem 2, we already studied the cases $\mathrm{G}$ is cyclic and finite, respectively. Therefore, let $\mathrm{G}$ now be noncyclic and infinite. Its structure is described in Theorem 4 which follows immediately from [6, p. 411, Theorem 3].

Theorem 4 If $\mathrm{G}$ is noncyclic and infinite but finitely generated, then it has cyclic subgroups $\mathrm{H}_{1}, \ldots, \mathrm{H}_{\mathrm{r}}$ of prime power order and a subgroup $\mathrm{H}_{0} \cong \mathbb{Z}^{n}$, $\mathrm{n} \geq 1$, such that

$$
\mathrm{G}=\mathrm{H}_{0} \oplus \mathrm{H}_{1} \oplus \cdots \oplus \mathrm{H}_{\mathrm{r}}=\mathrm{H}_{0} \oplus \mathrm{K} .
$$

These orders are unique. All decompositions (10) have the same number of summands of each order.

Applying our previous results, it is now easy to study maximal perpendicularities in G.

Theorem 5 Let $\mathrm{G}$ be as in Theorem 4 . If $\mathrm{n}>1$, then $\mathrm{G}$ has infinitely many maximal perpendicularities.

Proof. Decompose $\mathrm{H}_{0}$ as in (2) and define perpendicularities $\perp_{0}$ and $\perp_{0}^{\prime}$ in $\mathrm{H}_{0}$ as in (5). By Lemma 2, a maximal perpendicularity in $\mathrm{H}_{0}$ cannot contain both of them. Therefore, regarding them also as relations in G, a maximal perpendicularity in $\mathrm{G}$ cannot either contain both of them. Because there are infinitely many $\perp_{0}$ 's, the claim follows.

The proof of the next theorem is very similar to that of Theorem 3. Actually, the proof applies also when one subgroup is infinite (but cyclic).

Theorem 6 Let $\mathrm{G}$ be as above. If $\mathrm{n}=1$ and $|\mathrm{K}|$ is square-free, then $\mathrm{G}$ has a unique maximal perpendicularity.

By the similarity between the above results and those in Section 3, we present an analogy to Conjecture 2 .

Conjecture 3 Let $\mathrm{G}$ be as above. The following conditions are equivalent:

(a) G has a unique maximal perpendicularity,

(b) $\mathrm{n}=1$ and $|\mathrm{K}|$ is square-free, 
(c) (10) is unique (up to the ordering of the $\mathrm{H}_{\mathrm{i}}$ 's).

Applying an analogous argument as in the proof of Proposition 5, we also get the following proposition.

Proposition 6 Let $\mathrm{G}$ be as above. If $\mathrm{G}$ has a unique maximal perpendicularity, then (10) is unique.

\section{$5 \quad \mathrm{G} \cong \mathbb{Z} \oplus \mathbb{Z} \oplus \cdots$}

Now, let $G \cong \mathbb{Z} \oplus \mathbb{Z} \oplus \cdots$ (i.e., the set of infinite integer sequences with only finitely many nonzero terms). We begin the examination of this case by recording a result corresponding to Theorem 1.

Theorem 7 There are infinitely many maximal perpendicularities in $\mathrm{G} \cong$ $\mathbb{Z} \oplus \mathbb{Z} \oplus \cdots$.

Proof. Analogously to (1), choose $g_{1}, g_{2}, \cdots \in G$ such that $g_{1}=(1,0,0, \ldots)$ and

$$
g_{i}=\left(\gamma_{i 1}, \ldots, \gamma_{i, i-1}, 1,0,0, \ldots\right), \quad i=1,2, \ldots,
$$

and $g_{1}^{\prime}, g_{2}^{\prime}, \ldots$ similarly. A simple modification of the proof of Theorem 1 applies.

Let $a, b \in G$. Write them as

$$
a=\alpha_{1} g_{1}+\alpha_{2} g_{2}+\ldots, \quad b=\beta_{1} g_{1}+\beta_{2} g_{2}+\ldots
$$

Analogously to (7), we define

$$
\mathrm{a} \perp_{1} \mathrm{~b} \Longleftrightarrow \alpha_{1} \beta_{1}+\alpha_{2} \beta_{2}+\cdots=0 .
$$

(The sum is finite, because only finitely many $\alpha_{i}$ 's and $\beta_{i}$ 's are nonzero.) Analogously to Conjecture 1, we state as follows.

Conjecture 4 Let $\mathrm{G}$ be as in Theorem 7. A perpendicularity in $\mathrm{G}$ is maximal if and only if it is of the form (11).

The question about the cardinality of the set of all perpendicularities in $\mathrm{G} \cong$ $\mathbb{Z}^{\mathfrak{n}}, \mathrm{n}>1$, remained open in Proposition 4 since the answer depends on Conjecture 1. However, we can solve this question in the case of $G=\mathbb{Z} \oplus \mathbb{Z} \oplus \cdots$. 
Proposition 7 Let $\mathrm{G}$ be as above. The set of its maximal perpendicularities has cardinality $2^{\mathrm{x}_{0}}$.

Proof. Let $S$ denote the set of maximal perpendicularities in $\mathrm{G}$. The set of all possible sequences $\left(g_{1}, g_{2}, \ldots\right)$ has cardinality $2^{\aleph_{0}}$. Therefore, $|S| \geq 2^{\aleph_{0}}$.

On the other hand,

$$
\mathrm{G}=A_{0} \cup A_{1} \cup A_{2} \cup \cdots,
$$

where $A_{0}=\{(0,0, \ldots)\}$ and

$$
A_{i}=\left\{\left(x_{0}, x_{1}, \ldots, x_{i}, 0,0, \ldots\right) \mid x_{0}, \ldots, x_{i} \in \mathbb{Z}, x_{i} \neq 0\right\}, \quad i=1,2, \ldots .
$$

Clearly, $\left|A_{1}\right|=\left|A_{2}\right|=\cdots=\aleph_{0}$. Hence, $|\mathrm{G}|=\aleph_{0}$ and, further, $|\mathrm{G} \times \mathrm{G}|=\mathbf{\aleph}_{0}$. This implies that the cardinality of the set of all binary relations in $G$ is $2^{\alpha_{0}}$. Consequently, $|S| \leq 2^{\mathrm{x}_{0}}$ and the claim follows.

\section{$6 \quad \mathrm{G}=\left(\mathbb{Q}_{+}, \cdot\right)$}

As a sequel to the previous section, we consider the multiplicative group $\mathbb{Q}_{+}$ of the set of positive rational numbers. Let $\mathbb{P}$ denote the set of primes. Every $x \in \mathbb{Q}_{+}$can be uniquely expressed as

$$
x=\prod_{p \in \mathbb{P}} p^{v(p, x)},
$$

where $v(p, x) \in \mathbb{Z}$ for each $p \in \mathbb{P}$, and only finitely many of them are nonzero. For example,

$$
\begin{array}{r}
v(2,45)=0, v(3,45)=2, v(5,45)=1, v(7,45)=v(11,45)=\cdots=0, \\
v(2,1)=v(3,1)=\cdots=0, \\
v\left(2, \frac{8}{25}\right)=3, v\left(3, \frac{8}{25}\right)=0, v\left(5, \frac{8}{25}\right)=-2, v\left(7, \frac{8}{25}\right)=v\left(11, \frac{8}{25}\right)=\cdots=0 .
\end{array}
$$

If the nonzero values of $v(p, x)$ are $v\left(p_{1}, x\right)=v_{1}, \ldots, v\left(p_{k}, x\right)=v_{k}$, then (12) reads

$$
x=p_{1}^{v_{1}} \cdots p_{k}^{v_{k}} .
$$

Define the mapping $\mathrm{F}: \mathbb{Q}_{+} \rightarrow \mathbb{Z} \oplus \mathbb{Z} \oplus \cdots$ by $\mathrm{F}(\mathrm{x})=(\vee(2, x), v(3, x), \ldots)$. For example

$$
\begin{gathered}
F(45)=F\left(2^{0} \cdot 3^{2} \cdot 5^{1} \cdot 7^{0} \ldots\right)=(0,2,1,0,0, \ldots), \\
F(1)=F\left(2^{0} \cdot 3^{0} \cdots\right)=(0,0, \ldots) \\
F\left(\frac{8}{25}\right)=F\left(2^{3} \cdot 3^{0} \cdot 5^{-2} \cdot 7^{0} \ldots\right)=(3,0,-2,0,0, \ldots) .
\end{gathered}
$$


It is easy to see that $F$ is an isomorphism. Thus all results of Section 5 are valid in $\mathbb{Q}_{+} ;$see also $[5$, Section 5$]$.

\section{$7 \quad \mathrm{G}=\left(\mathbb{R}^{\mathrm{n}},+\right), \mathrm{n}>1$}

Finally, let us consider $G=\left(\mathbb{R}^{n},+\right), n>1$. If $a=\left(\alpha_{1}, \ldots, \alpha_{n}\right), b=\left(\beta_{1}, \ldots, \beta_{n}\right) \in$ $\mathrm{G}$, we can define, analogously to (5), the perpendicularity $\perp_{0}$ :

$$
\mathrm{a} \perp_{0} \mathrm{~b} \Longleftrightarrow \forall i \in N: \alpha_{i}=0 \vee \beta_{i}=0 .
$$

However, we cannot define, analogously to (7), the perpendicularity $\perp_{1}$ :

$$
\mathrm{a} \perp_{1} \mathrm{~b} \Longleftrightarrow \alpha_{1} \beta_{1}+\cdots+\alpha_{n} \beta_{\mathrm{n}}=0,
$$

because we are allowed to use only addition (and multiplication by an integer) in this group. Therefore it is reasonable to regard $\mathbb{R}^{n}$ as a vector space rather than as a group, but perpendicularity in a vector space is beyond our scope. However, we take a small step to it and, more generally, to perpendicularity in a module.

Let $M \neq\{0\}$ be a module over a ring $R$. We say that a relation $\perp$ in $M$ is a perpendicularity in $M$ if it satisfies (A1)-(A5) and

(A6) $\forall \mathrm{a}, \mathrm{b} \in \mathrm{M}, \gamma \in \mathrm{R}: \mathrm{a} \perp \mathrm{b} \Rightarrow \mathrm{a} \perp \gamma \mathrm{b}$.

Since an Abelian group $G$ is a module over $\mathbb{Z}$, a perpendicularity in $G$ is also a perpendicularity in this module.

We define in the vector space $V=\mathbb{R}^{n}, n>1$, the Euclidean inner product

$$
\langle a, b\rangle=\alpha_{1} \beta_{1}+\cdots+\alpha_{n} \beta_{n} .
$$

The relation $\perp_{1}$ :

$$
\mathrm{a} \perp_{1} \mathrm{~b} \Longleftrightarrow\langle\mathrm{a}, \mathrm{b}\rangle=0
$$

is clearly a perpendicularity in $\mathrm{V}$. We show that it is maximal.

We proceed by contradiction. Suppose that $\perp_{1}$ is a proper subset of a perpendicularity $\perp$. Then there are $a, b \in V$ with $a \perp b$ and $a \not \perp_{1} b$. Since the orthogonal complement $\{a\}^{\perp_{1}}$ is an $(n-1)$-dimensional subspace of $V$, it is spanned by a linearly independent set $S=\left\{c_{1}, \ldots, c_{n-1}\right\}$. Here $a \perp_{1} c_{1}, \ldots, c_{n-1}$ and, because $\perp_{1} \subset \perp$, also $a \perp c_{1}, \ldots, c_{n-1}$. Since $b \notin\{a\}^{\perp_{1}}=$ span $S$, the set $S_{b}=S \cup\{b\}$ is linearly independent; so it spans $V$. Now $a \perp c_{1}, \ldots, c_{n-1}, b$; 
hence $\mathrm{a} \in\left(\mathrm{S}_{\mathrm{b}}\right)^{\perp}=\mathrm{V}^{\perp}=\{0\}$, which implies that $\mathrm{a}=0$. But then $\mathrm{a} \perp_{1} \mathrm{~b}$, contradicting $a \not \not_{1} b$.

Let $\mathrm{Q}$ be a real symmetric positive definite $n \times n$ matrix. Define in $\mathrm{V}$ the inner product

$$
[a, b]=\langle Q a, b\rangle .
$$

The above proof applies also to the perpendicularity $\perp^{\prime}$ :

$$
\mathrm{a} \perp^{\prime} \mathrm{b} \Longleftrightarrow[\mathrm{a}, \mathrm{b}]=0 ;
$$

so $\perp^{\prime}$ is maximal. Conversely, we conjecture that all maximal perpendicularities in $\mathrm{V}$ are obtained in this way.

\section{Summary}

For $G \cong \mathbb{Z}^{\mathrm{n}}, \mathrm{G}$ finite, $\mathrm{G}$ finitely generated, and $\mathrm{G}=\mathbb{Z} \oplus \mathbb{Z} \oplus \cdots$, we were able to only partially answer the question how many maximal perpendicularities $\mathrm{G}$ has. Nevertheless, these results may assist us in characterizing all maximal perpendicularities in the case $\mathrm{G} \cong \mathbb{Z}^{\mathfrak{n}}$ or $\mathrm{G}=\mathbb{Z} \oplus \mathbb{Z} \oplus \cdots$ (Conjectures 1 and 4 ) or, in the case $\mathrm{G}$ is finite or finitely generated, to typify those Abelian groups that have a unique maximal perpendicularity (Conjectures 2 and 3). However, more effort is needed to know whether our suppositions were correct or not. In studying $\mathbb{R}^{n}$, we encountered perpendicularity in a vector space and, more generally, in a module. This topic is interesting for further research.

\section{Acknowledgement}

The authors wish to thank the anonymous referee for comments that helped to improve this paper.

\section{References}

[1] F. Bachmann, Aufbau der Geometrie aus dem Spiegelungsbegriff (German), Springer, 1959 (2nd revised edition 1973).

[2] G. Davis, Rings with orthogonality relations, Bull. Austral. Math. Soc., 4 (1971), 163-178.

[3] G. Davis, Orthogonality relations on abelian groups, J. Austral. Math. Soc., 4 (1975), 173-179. 
[4] P. Haukkanen, J. K. Merikoski, T. Tossavainen, Axiomatizing perpendicularity and parallelism, J. Geom. Graph., 15 (2011), 129-139.

[5] P. Haukkanen, M. Mattila, J. K. Merikoski, T. Tossavainen, Perpendicularity in an Abelian group, Internat. J. Math. Math. Sci., 2013, Art. ID 983607,8 pp.

[6] W. K. Nicholson, Introduction to Abstract Algebra, Second Edition, John Wiley, 1999.

[7] A. I. Veksler, Linear spaces with disjoint elements and their conversion into vector lattices (Russian), Leningrad. Gos. Ped. Inst. Učen. Zap., $\mathbf{3 2 8}$ (1967), 19-43.

Received: May 24, 2016 